\title{
Alzheimer's Disease: Parsing the Pathways Leading to the Disease Based on the Spirochete/Biofilm Hypothesis
}

Herbert B. Allen *

Department of Dermatology, Drexel University College of Medicine, Philadelphia, PA, USA; E-Mail: hba25@drexel.edu

* Correspondence: Herbert B. Allen; E-Mail: hba25@drexel.edu

Academic Editor: Md. Golam Sharoar

Special Issue: Aging and Alzheimer's Disease

OBM Geriatrics

2020, volume 4, issue 1

doi:10.21926/obm.geriatr.2001107
Received: December 31, 2019

Accepted: February 17, 2020

Published: February 19, 2020

\begin{abstract}
A pathway which highlighted spirochetes (Borrelia burgdorferi and dental treponemes) that made biofilms which led to Alzheimer's disease has recently been promulgated. All the Alzheimer's disease, on which this pathway was based, had been specifically confirmed both clinically and pathologically. This current work will show putative and plausible individual pathways that were studied. First and foremost, intracellular spirochetes make biofilms and concurrently make beta amyloid; this has been shown in vitro in pure culture and in vivo. The beta amyloid together with tau protein leads to hyperphosphorylated tau that leads to neurofibrillary tangles and dendrite disintegration. Extracellular biofilms lead to activation of the innate immune system (similar to other chronic diseases), and this together with its major pathway (MyD88) leads to the production of beta amyloid. Many drugs and environmental states interact with that pathway and generally lead to further disease progression. (These drugs such as haloperidol, and environmental states such as hyperosmolality have been known to cause worsening of the disease.) Few things lead to reversal of the pathway, though L-serine stands out among them. All these pathways would not even exist or be activated were the spirochetes not present. The above hypothesis is
\end{abstract}

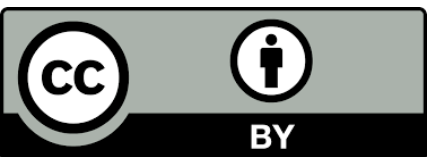

(C) 2020 by the author. This is an open access article distributed under the conditions of the Creative Commons by Attribution License, which permits unrestricted use, distribution, and reproduction in any medium or format, provided the original work is correctly cited. 
based on observed findings from patients with Alzheimer's disease and from pathways known to be generated from those findings.

\section{Keywords}

Alzheimer's disease; beta amyloid; tau tangles; Toll-like receptor 2

\section{Definition: Biofilm}

A biofilm is a community of microbial organisms encased in a slime coating that arises in response to environmental or antimicrobial stressors. The biofilm consists of extracellular polysaccharides and a proteinaceous infrastructure of amyloid. The polysaccharides stain histologically with periodic acid Schiff, and the amyloid stains with Congo red. Other components of biofilms include smaller amounts of DNA, RNA, water, lipids, and exporter cells. Biofilms generally form by quorum sensing, and the organisms have many genes for this for this to occur. They also preferentially attach to other substrates such as catheters. Biofilms have attachment sites for Toll-like receptor 2 which, in turn, utilizes the Myeloid Differentiation 88 pathway to attempt to inactivate the microbes. (Chronic) diseases result when that activity attacks surrounding tissue and generates deleterious activity; further, biofilms are often polymicrobial.

\section{Introduction}

A pathway to Alzheimer's disease (AD) has recently been delineated that, in essence, described spirochetes making biofilms that subsequently led to AD [1]. (Figure 1) This hypothesis was based on a template that had shown AD to be similar to general paresis (GP) of the insane of tertiary syphilis in regard to both the clinical and pathological presentations [2]. This similarity showed the major pathological components (spirochetes, senile plaques, neurofibrillary tangles, and beta amyloid [Abeta]) were present in both AD and GP. Also noted, in both, were granulovacuolar degeneration, neuronal loss, and tissue atrophy $[2,3]$.

\section{Spirochetes $\Longrightarrow$ Biofilms $\Longrightarrow$ Alzheimer's disease}

Figure 1 Overall pathway.

The spirochetal biofilms have been shown to be present both intra and extra-cellularly where they produce dramatically different effects that have been demonstrated both pathologically and microbiologically [4]. During the formation of the biofilms by the spirochetes, the spirochetes have also been shown, in pure culture, to make beta amyloid precursor protein (ABPP) and Abeta simultaneously with the biofilm [5]. Abeta has been demonstrated pathologically to be both intracellular and extracellular [4]. Abeta interacts with tau protein; and, this leads to tau hyperphosphorylation ( $p$-tau), localized $\mathrm{Ca} 2+$ elevation, tau missorting into dendrites, and destruction of microtubules and spines $[6,7]$. One isoform of $p$-tau has actually been shown to be protective of dendrites [8]. The toxic p-tau leads to disintegration of the neuronal dendrites 
(because they are no longer stabilized by ordinary tau protein), and it also leads to the formation of neurofibrillary tangles (NFT) [2]. Spirochetes have been found in NFT [2]. This is a very important pathway because of the neuronal destruction that eventuates. (Figures 2 and 3) This was foreshadowed when transgenic mice had their cognitive decline ameliorated by reduction of Abeta and tau, but not Abeta alone [9].

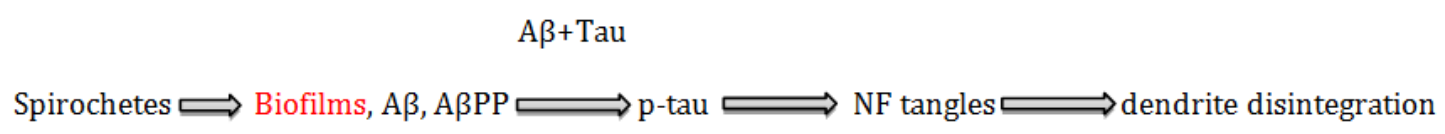

Figure 2 Intracellular pathway.

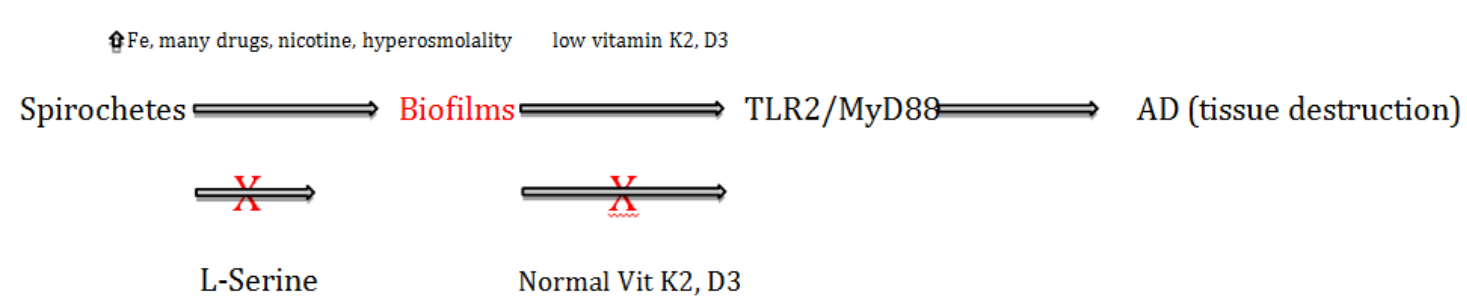

Figure 3 Extracellular pathway.

Continuing with the various pathways leading to dementia, intracellular biofilms within the hippocampal neurons likely form with "quorum sensing" [10]. The quorum requires 10 microbes in any direction to begin forming a biofilm [11]. The community formed in this manner "fits" in the cytoplasm of the neuron $[12,13]$. If the space is not large enough, the organisms remain planktonic without a biofilm developing. It is unlikely that biofilm dispersers such as nicotine have much impact on intracellular biofilms because only $30 \%$ (at most) crosses the cell membrane [14, 15]. It is possible that hypermosmolality impacts intracellular biofilms because of the osmosis of fluid from inside to outside the cell would stress the biofilm [16]. A neurotoxin, beta methyl amino alanine (BMAA), may enter into the intracellular space and impact biofilms because it triggers formation of neurofibrillary tangles and Abeta deposits in the brains of Vervet monkeys [17].

Extracellular biofilms in the brain behave similarly to extracellular biofilms in other diseases [18]. Namely, they upregulate Toll-like receptor 2 (TLR2) of the innate immune system, and they are also subject to both biofilm dispersers and aggregators as well as environmental compounds and states [19]. Many drugs are biofilm dispersers (furans, piperidines, pyrroles, thiophenes, and rifampin) and thus cause disruption and subsequent new formation of more biofilms [19]. (Figure 4) This is similar to the extrusion of exporter cells as a natural occurrence in a biofilm. An example of a pharmacological biofilm disperser causing severe difficulty is Haloperidol (a piperidine) which leads to a $200 \%$ increase in death when administered to AD patients [20].

TLR2 that is upregulated by extracellular biofilms, even those that are created by gram negative organisms, utilizes the myeloid differentiation 88 pathway (MyD88) to inactivate microbes, but is unable to penetrate the biofilm, so the spirochetes remain safely ensconced inside [21, 22].

The TLR2/MyD88 generates NFkB and TNFa that utilize another pathway whereby NFkB catalyzes beta amyloid converting enzyme that, in turn, catalyzes $\beta$ and $\gamma$ secretase that cleaves off the terminal portions of ABPP to form Abeta [21]. Thus, extracellular Abeta is generated by this 
sequence as well as that which arises when the $p$-tau neurons degenerate. This latter leads to the equivalent of exporter cells being extruded from a mature biofilm. (Figure 5)

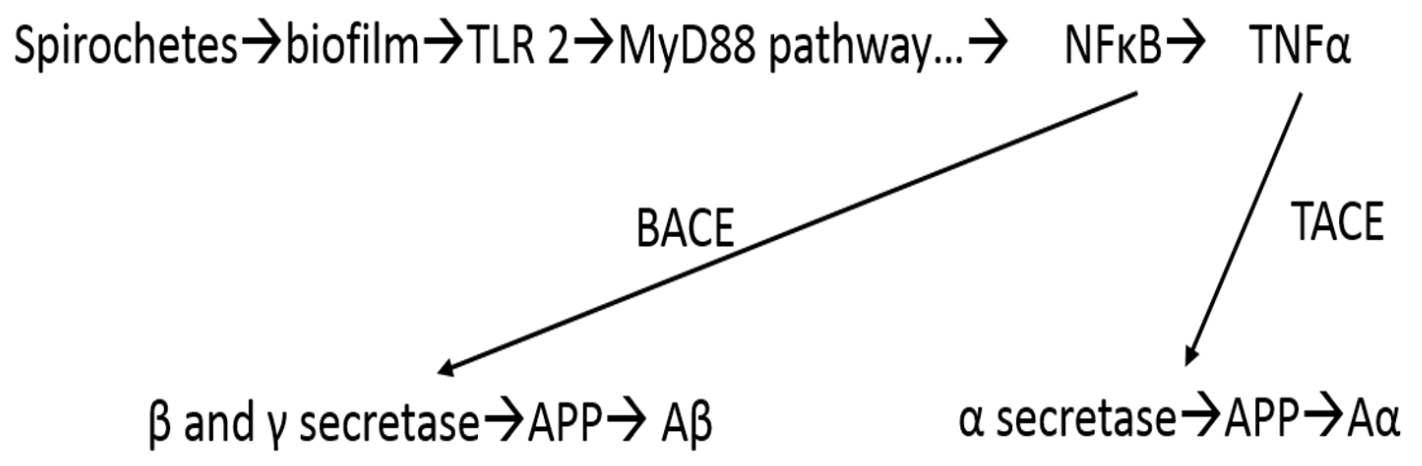

Figure 4 Pathway to Abeta from spirochetes/biofilm/immune system. From J Alzheimers Dis 2016; 53: 1271-1276.

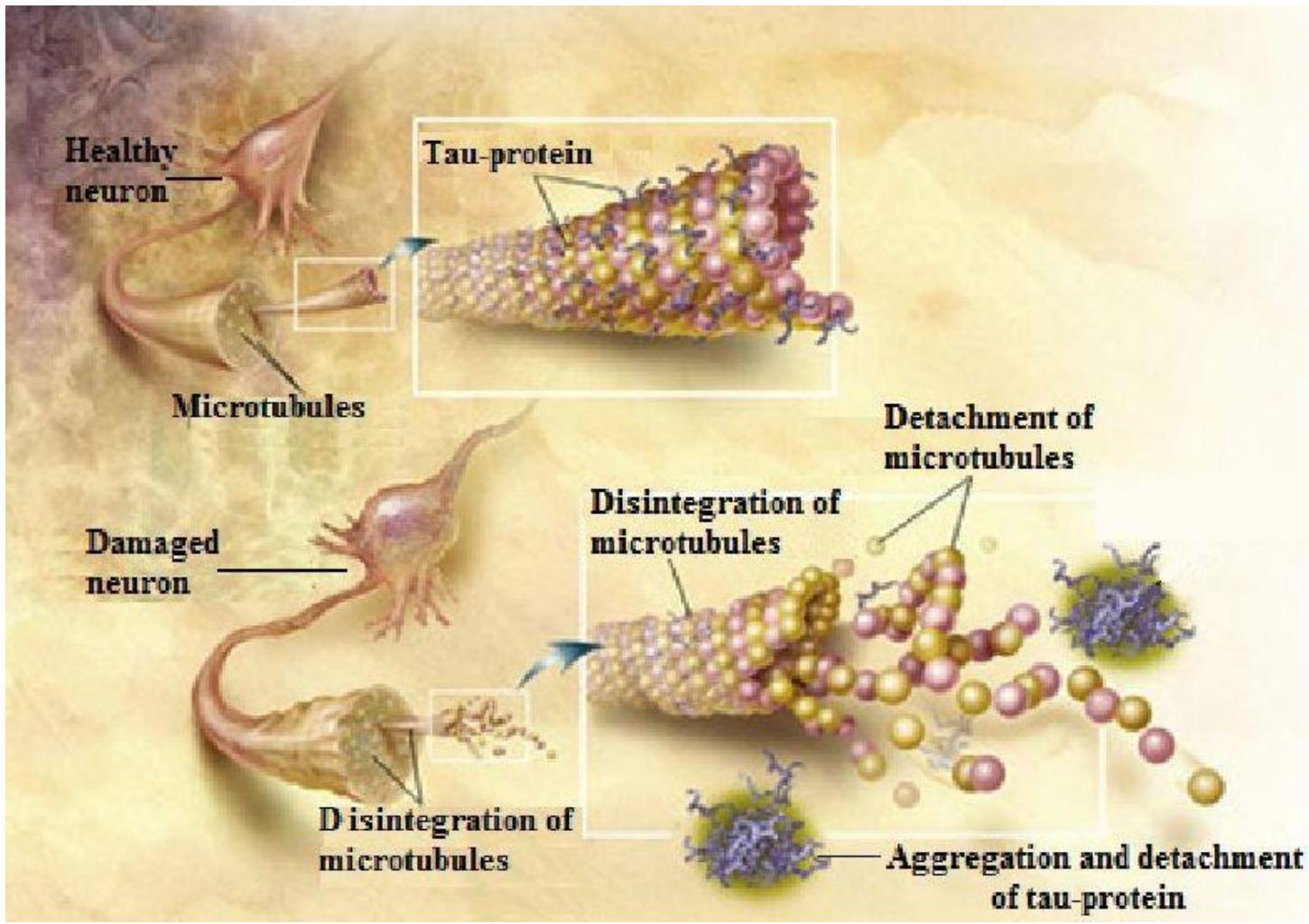

Figure 5 Disintegration of neuronal dendrites from $p$-tau. Schematic from ADEAR AD education and referral center.

Biofilms form more readily when the surrounding serum contains low vitamin $E$ and elevated serum iron [23]. The opposite occurs in a serum rich with L-serine which inhibits quorum sensing [17]. Caffeine may also be a mild quorum sensing inhibitor [24]. Vitamins K2 and D3, if low, cause upregulation of TLR2 leading to consequences already discussed; normal to slightly elevated K2 and D3 do the opposite and lead to a lower impact of TLR2 [25, 26]. (Figure 4)

These various pathways would not exist if the spirochetes initiating them were killed prior to the formation of biofilms. The Borrelial and dental spirochetes are susceptible to penicillin, and 
penicillin derivatives, and a once yearly course of that antibiotic would seem sufficient to carry this out. Azithromycin would be an alternative for the penicillin allergic. The course derives from the treatment of syphilis, the absolute prototype for AD. Treatment with penicillin anytime prior to tertiary is curative. The yearly course could be considered like a "vaccine", necessary because of the constant seeding of dental spirochetes in the brain. This would likely reduce resistance as well because, once organisms are in a biofilm, they pass resistance genes horizontally. The dental seeding is unlike syphilis or Lyme disease where the exposure is likely to be a one-time event. Such as approach seems rational until such time as a serological test (or other) is developed which can predict $A D$, just as the RPR predicts tertiary syphilis [27]. The serologic test has been foremost in the disappearance of tertiary syphilis. Another chronic biofilm disease, leprosy, has nearly disappeared with the administration of Dapsone and rifampicin (a biofilm disperser) [28]. Such a protocol would be unsuccessful in $A D$ because it would be rendered too late in the course of the disease [29].

Other efforts have presented these pathways with a singular focus $[3,5,10,15,17]$. This work attempts to align each of them into an overall pathway to the disease. It seems apparent that the intracellular occurrences are more important inasmuch as they lead to destruction of the neurons. All this is shown to be a result of a chronic infection which spirochetes and their biofilms play a leading role. This conforms to other chronic infections in which microbes and their biofilms cause the diseases [18].

\section{Author Contributions}

Herbert B. Allen was the sole author.

\section{Competing Interests}

The author has declared that no competing interests exist.

\section{References}

1. Allen HB, Allawh RM, Goyal K. A pathway to Alzheimer's disease. J Curr Neurobiol. 2018; 9: 29-32.

2. Miklossy J. Historic evidence to support a causal relationship between spirochetal infections and Alzheimer's disease. Front Aging Neurosci. 2015; 7: 46.

3. Macdonald AB. Alzheimer's neuroborreliosis with trans-synaptic spread of infection and neurofibrillary tangles derived from intraneuronal spirochetes. Med Hypotheses. 2007; 68: 822-825.

4. Allen HB. The essential role of biofilms in Alzheimer's disease. Microbiol Infect Dis. 2019; 3: 13.

5. Miklossy J. Bacterial amyloid and DNA are important constituents of senile plaques: Further evidence of the spirochetal and biofilm nature of senile plaques. J Alzheimers Dis. 2016; 53: 1459-1473.

6. Zempel $H$, Thies $E$, Mandelkow E, Mandelkow EM. A $\beta$ oligomers cause localized Ca2 elevation, missorting of endogenous tau into dendrites, tau phosphorylation, and destruction of microtubules and spines. J Neurosci. 2010; 30: 11938-11950. 
7. Iqbal K, Alonso AC, Chen S, Chohan MO, El-Akkad E, Gong CX, et al. Tau pathology in Alzheimer disease and other tauopathies. Biochim Biophys Acta. 2005; 1739: 198-210.

8. Dolan PJ, Johnson GVW. The role of tau kinases in Alzheimer's disease. Curr Opin Drug Dosov Devel. 2010; 13: 595-603.

9. Oddo S, Vasilevko V, Cascano A, Kitazawa M, Cribbs DH, LaFerla FM. Reduction of soluble $A \beta$ and tau, but not soluble $A \beta$ alone, ameliorates cognitive decline in transgenic mice with plaques and tangles. J Biol Chem. 2006; 281: 39413-39423.

10. Rutherford ST, Bassler BL. Bacterial quorum sensing: Its role in virulence and possibilities for its control. Cold Spring Harb. 2012; 2: a012427.

11. Allen HB, Goyal K, Ogrich L, Joshi S. Biofilm formation by Malassezia Furfur/Ovale as a possible mechanism of pathogenesis in tinea versicolor . J Clin Exp Dermatol Res. 2015; 6: 311.

12. Allen HB, Allawh R, Touati A, Katsetos C, Joshi SG. Alzheimer's disease: The novel finding of intracellular biofilms. J Neuroinfect Dis. 2017; 8: 247.

13. Allen HB, Allawh RM, Cusack CA, Joshi SG. Alzheimer's disease: Intracellular beta amyloid completes the irreversible pathway from spirochetes to biofilms to beta amyloid to hyperphosphorylated tau protein. J Neuroinfect Dis. 2018; 9: 276.

14. Le Houezec J. Role of nicotine pharmacokinetics in nicotine addiction and nicotine replacement therapy: A review. Int J Tuberc Lung Dis. 2003; 7: 811-819.

15. Allen HB, Joshi SG. Nicotine and Alzheimer's disease: Mechanism for how the fog of smoke increases the fog of dementia. J Neuroinfect Dis. 2016; 7: 237.

16. Allen HB, Husienzad L, Joshi SG. The impact of diabetes on Alzheimer's disease. J Alzheimers Dis. 2016.

17. Cox PA, Davis DA, Mash DC, Metcalf JS, Banack SA. Dietary exposure to an environmental toxin triggers neurofibrillary tangles and amyloid deposits in the brain. Proc R Soc B. 2016; 283: 1-10.

18. Allen HB, Cusack CA, Joshi SG. The impact of biofilms in Alzheimer's disease compared to other diseases in which they play a role. Int J Neurobiol. 2019; 1: 115.

19. Allen HB, Allawh RM, Ilyas EN, Miklossy J, Joshi SG. Alzheimer's disease: Possible mechanisms for worsening of the disease. Curr Neurobiol. 2018; 9: 59-65.

20. Huybrechts KF, Gerhard T, Crystal S, Olfson M, Avorn J, Levin R, et al. Differential risk of death in older residents in nursing homes prescribed specific antipsychotic drugs: Population based cohort study. BMJ. 2012; 344: e977.

21. Allen HB. Alzheimer's disease: Assessing the role of spirochetes, biofilms, the immune system, and amyloid- $\beta$ with regard to potential treatment and prevention. J Alzheimers Dis. 2016; 53: 1271-1276.

22. Tukel C, Wilson RP, Nishimori M, Pezeshki M, Chromy BA, Baumier AG. Responses to amyloids of microbial and host origin are mediated through Toll-like receptor 2. Cell Host Microbe. 2009; 6: 45-53.

23. Weinberg ED, Miklossy J. Iron withholding: A defense against disease. J Alzheimers Dis. 2008; 13: 451-463.

24. Cao C, Loewenstein DA, Lin X, Zhang C, Wang L, Duara R, et al. High blood caffeine levels in $\mathrm{MCl}$ linked to lack of progression to dementia. J Alzheimers Dis. 2012; 30: 559-572.

25. https://www.patreon.com/posts/endotoxin-part-7130268 
26. Gezen-Ak D, Yılmazer S, Dursun E. Why vitamin D in Alzheimer's disease? The hypothesis. J Alzheimers Dis. 2014; 40: 257-269.

27. Allen HB. Alzheimer's disease: Future efforts to thwart memory loss. Dementia. 2019; 256: 2195-2196.

28. Allen HB, Moschella SL. The role of rifampin in leprosy: leprosy through a new lens. JAMA Dermatol. 2017; 153: 261-262.

29. Allen HB, Allawh RM, Ilyas EN, Joshi SG. Bioethical challenges arising from the microbiology and pathology of Alzheimer's disease. Curr Neurobiol. 2018: 9: 26-28.

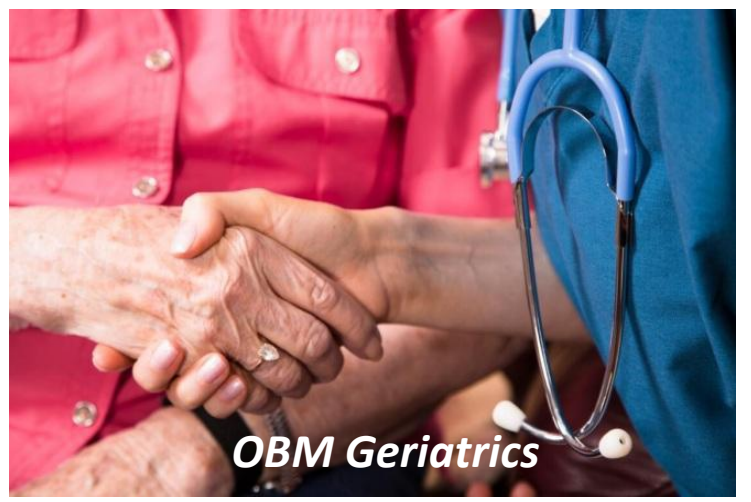

Enjoy OBM Geriatrics by:

1. Submitting a manuscript

2. Joining in volunteer reviewer bank

3. Joining Editorial Board

4. Guest editing a special issue

For more details, please visit:

http://www.lidsen.com/journals/geriatrics 